\title{
Driver Fatigue Detection and Alert System using Non- Intrusive Eye and Yawn Detection
}

\author{
Pranali Awasekar \\ Computer Engineering \\ K.J. Somaiya College of \\ Engineering, Vidyavihar \\ Mumbai, India
}

\author{
Menaka Ravi \\ Computer Engineering \\ K.J. Somaiya College of \\ Engineering, Vidyavihar \\ Mumbai, India
}

\author{
Shivani Doke \\ Computer Engineering \\ K.J. Somaiya College of \\ Engineering, Vidyavihar \\ Mumbai, India
}

\author{
Zaheed Shaikh \\ Professor, Computer \\ Engineering \\ K.J. Somaiya College of \\ Engineering, Vidyavihar \\ Mumbai, India
}

\begin{abstract}
Driver fatigue is one the leading causes of car accidents in the world. Detecting drowsiness and alerting the driver is the easiest way to prevent mishaps. The purpose of this paper is to develop a fatigue detection and alert system. This system works by analyzing the eye closure duration and yawn frequency of the driver and alerting the driver by activating LEDs, buzzers and sending warning message to his emergency contacts. The alerts are divided into three stages of severity to take action accordingly. Facial features for determining alertness were obtained by using a camera capturing the face of the driver. The system can monitor the driver's eyes to detect early stages of sleep as well as short periods of sleep lasting 3 to 4 seconds. The application is implemented on a Raspberry Pi minicomputer with a NoIR camera, making the system economical and portable. The system not only provides an effective way to detect fatigue but also provides many forms of alerts to control the situation and compel the driver to take a break.
\end{abstract}

\section{General Terms}

Image Processing.Wireless connectivity.

\section{Keywords}

fatigue detection, road safety, image processing, early warning, facial landmark, Haar cascade, regression trees, Bluetooth Low Energy, GPS.

\section{INTRODUCTION}

Ideally, each individual needs between seven to eight hours of quality sleep each night. In worst cases, drivers with sleep deprivation risk nodding off; yet even minor fatigue can impair their reaction time and decision making abilities while driving, thus increasing the risk of accident. According to fatigue statistics [1][2], if a driver falls asleep for just four seconds while travelling at a speed of $100 \mathrm{~km} / \mathrm{h}$ the car will have gone 111 meters without a driver in control. At high speed, the probability of a crash is high with a risk of death or severe injury. Fatigue is the sixth major killer in the world resulting in some 60 deaths and approximately 300 serious injuries each year [1][2]. Driver safety being of utmost importance and an expensive technology to incorporate, we have come up with a non-intrusive, economic and effective way to end this problem.

\section{LITERATURE SURVEY}

Most systems introduced so far lack the precision to detect the intensity of drowsiness and fatigue, moreover only the eye blink factor is considered to detect fatigue. In the real world, the eye blink rates may vary from person to person rendering the system ineffective or less accurate. This project aims to detect the exact phase of drivers fatigue using eye closure duration and yawn frequency and takes the necessary action depending on the urgency of the situation.

\subsection{Existing Systems}

The existing systems are based upon a number of metrics, like the deviations from lane position, movement of the steering wheel, physiological measurement , etc., However each of these have restrictions and cannot be deployed easily.

\subsubsection{Steering pattern monitoring}

This system [3] primarily uses steering input from an electric power steering system. Being a cost demanding project it is not feasible. This system requires advanced signal processing procedures for feature extraction, it computes 3 feature set in the time, frequency and state space domain to capture fatigue impaired steering patterns. Some car companies like, Nissan and Renault, have adopted SPMs but it works only in few situations. It is mainly because they are dependent on the geometric features of the road and not the kinetic characteristics of the vehicle [4].

\subsubsection{Standard Deviation of Lane Position}

The lane keeping quality is selected in this approach to indicate the behavior of the driver [5]. Adequate lane keeping is important for traffic safety, because extreme lane changing points out anomaly in driver behavior, which might be due to fatigue. Different aspects of lane maintaining quality are used when assessing the driving performance of fatigue for example the standard deviation of lateral position (SDLP), the number of lane departures, time to line crossing (TLC). This method has it disadvantages, it requires many sensors and the changes in lane can be due to quality of road edge. The driver behavior may differ from road to road, also remote monitoring is crucial in this method [4].

\subsubsection{Physiological measurement}

The relationship between physiological signals (electrooculogram (EoG), electrocardiogram (ECG), electromyogram (EMG), and electroencephalogram (EEG)) and driver fatigue has been studied by many researchers [6]. This method requires body sensors like heart rate, brain and muscle activity and skin conductance. Although this approach is reliable and accurate as physiological signals are very precise in comparison to other methods, it is intrusive, irritable and inconvenient for drivers during long travel hours.

\section{PROPOSED SYSTEM}

\subsection{Hardware Setup}

\subsubsection{Raspberry Pi and Camera}

A NoIR camera is mounted on the dashboard of the vehicle such that it is at a distance of two to three feet from the driver, ensuring that variations due to the height of driver or sitting 
position are incorporated. This positioning enables clear capture of the facial landmarks by the NoIR camera integrated with the Raspberry Pi module. The camera captures a 90 degree field of view. The reason a NoIR camera was chosen is because it enables image capture in broad daylight as well as very poorly lit conditions [7]. To assist image capture in complete darkness, as it may be required during night-time, a dim IR lamp may be used to illuminate the scene without causing disturbance to the driver. The system captures and processes images at the rate of 6-8 frames per second, a reasonable rate to ensure timely alerts.

\subsubsection{Android Connectivity}

The driver's Android smartphone can be used to send customized warnings to upto three emergency contacts in the form of SMS alerts. The smartphone must have our app installed which will connect it to the Raspberry $\mathrm{Pi}$ via bluetooth. Thus the smartphone can receive signals from the minicomputer when fatal drowsiness is detected, and in turn send out emergency messages to predefined contacts if the functionality is enabled.

\subsection{System Architecture}

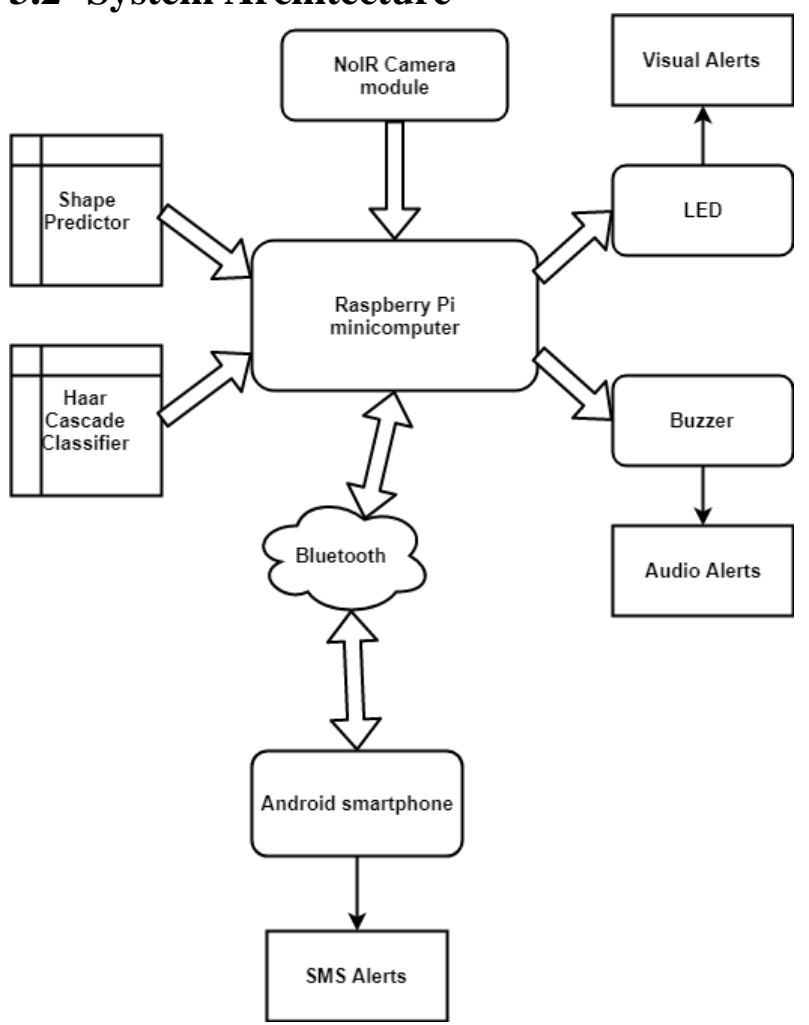

Fig 1: System architecture

\subsection{Working of the System}

The system can be broken down into the following phases-

\subsubsection{Image Capture and Fatigue Detection}

This step includes capturing the face of the driver frame by frame and subsequent facial landmark recognition, i.e, the recognition of the eye and mouth regions. Image capturing and processing takes place through the camera and Raspberry $\mathrm{Pi}$ module. This is followed by eye closure and yawn detection. Based on these two parameters, the system determines if the driver is drowsy or not.

\subsubsection{Alert System}

The local alerts beepers and LED lights are used to notify the driver in case the drowsy state is detected. The buzzer is adjusted to three levels of activation, with the increase in frequency of alerts for every stage that is detected. The sounding of the buzzer and blinking of the LEDs are designed to catch the attention of the driver and alert him/her of imminent sleep. When the highest stage of fatigue is detected, the Raspberry Pi sends a signal to the blutooth-connected smartphone. When this signal is received, the smartphone app generates an SMS message and sends it to the emergency contacts that the driver may have listed.

\section{METHODOLOGY}

\subsection{Abbreviations and Acronyms}

\subsubsection{Euclidean distance $(d)$}

It is the straight line distance between any two points in a Cartesian or Euclidean space [8]. It is given as-

$$
\begin{aligned}
\mathrm{d}(\mathrm{p}, \mathrm{q})=\mathrm{d}(\mathrm{q}, \mathrm{p}) & =\sqrt{\left(\mathrm{q}_{1}-\mathrm{p}_{1}\right)^{2}+\left(\mathrm{q}_{2}-\mathrm{p}_{2}\right)^{2}+\ldots+\left(\mathrm{q}_{\mathrm{n}}-\mathrm{p}_{\mathrm{n}}\right)^{2}} \\
& =\sqrt{\sum_{\mathrm{i}=1}^{\mathrm{n}}\left(\mathrm{q}_{\mathrm{i}}-\mathrm{p}_{\mathrm{i}}\right)^{2}}
\end{aligned}
$$

\subsubsection{Eye Aspect Ratio (EAR)}

Here it is defined as the ratio between the height and the width of the eye contour.

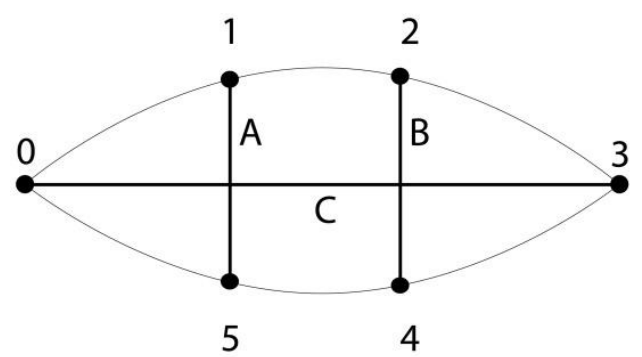

Fig 2: Eye region with reference coordinate points

$$
\begin{aligned}
& \mathrm{Ae}=\mathrm{d}(\text { eye[1], eye[5]) } \\
& \mathrm{Be}=\mathrm{d}(\text { eye[2], eye[4] }) \\
& \mathrm{Ce}=\mathrm{d}(\text { eye [0], eye [3] })
\end{aligned}
$$

$A_{e}$ and $B_{e}$ measure the vertical distance of the eye and $C_{e}$ calculates the horizontal dimensions of the eye.

$$
\mathrm{EAR}=\left(\mathrm{A}_{\mathrm{e}}+\mathrm{B}_{\mathrm{e}}\right) /\left(2.0 * \mathrm{C}_{\mathrm{e}}\right)
$$

\subsubsection{Eye Threshold $\left(T_{e}\right)$}

It is the threshold value of EAR. If the EAR in a frame falls below this value, the eye is considered to be closed.

$$
\text { Eye State }=\left\{\begin{array}{l}
\text { Closed, EAR }<T_{e} \\
\text { Open, EAR } \geq T_{e}
\end{array}\right.
$$

The value of Te was determined by trial and error, checking for different values of Te such that the system is correctly able to classify an instance of open or closed eyes. By this method we obtained an optimal value of Te as-

$$
\mathrm{Te}=0.3
$$




\subsubsection{Mouth Aspect Ratio (MAR)}

Here it is defined as the ratio between the height and the width of the mouth contour.

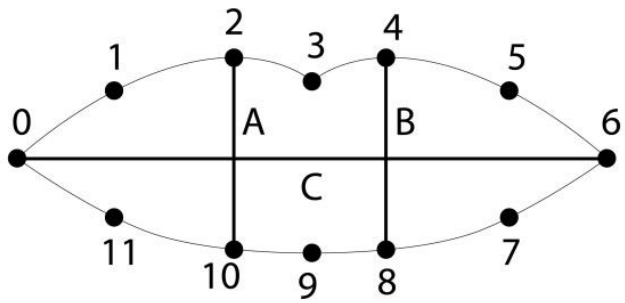

Fig 3: Mouth region with reference coordinate points

$$
\begin{aligned}
& \mathrm{Am}=\mathrm{d}(\text { mouth[2], mouth[10]) } \\
& \mathrm{Bm}=\mathrm{d}(\text { mouth[4], mouth[8]) } \\
& \mathrm{Cm}=\mathrm{d}(\text { mouth[0], mouth[6]) }
\end{aligned}
$$

$\mathrm{Am}$ and $\mathrm{Bm}$ measure the vertical distance of the eye and $\mathrm{Cm}$ calculates the horizontal dimensions of the eye.

$$
\mathrm{MAR}=(\mathrm{Am}+\mathrm{Bm}) /(2.0 * \mathrm{Cm})
$$

\subsubsection{Mouth Threshold $\left(T_{m}\right)$}

It is the threshold value of MAR. If the MAR in a frame exceeds this value, the mouth is considered to be in a yawning state.

$$
\text { Mouth State }=\left\{\begin{aligned}
\text { Yawn, } & \text { MAR }<T_{m} \\
\text { Closed } / \text { Talking, } & M A R \geq T_{m}
\end{aligned}\right.
$$

The value of $T_{m}$ was determined by trial and error, checking for different values of $T_{m}$ such that the system is correctly able to classify an instance of yawning and closed mouth. In determining the optimal threshold for yawning mouth, a problem of detecting false positives was faced wherein the driver may have his/her mouth open while talking. This must not be identified as a state of yawning. Since human mouth opens much wider over a number of consecutive frames while yawning as compared to talking, the threshold is selected in such a way that it is high enough to ignore open mouth while talking under normal conditions [9]. By this method we obtained an optimal value of $\mathrm{T}_{\mathrm{m}}$ as-

$$
\mathrm{T}_{\mathrm{m}}=0.9
$$

\subsection{Fatigue Detection}

When the system is started, it begins to capture the driver's frontal video, which is then processed frame by frame. Each frame or individual image is processed to first identify the face of the driver. Face detection is accomplished by using a Haar cascade classifier that is trained to recognize human faces [10].

On identifying the face rectangle region, the next step is to determine the location of key facial landmarks. This detection is carried out by using an ensemble of regression trees [11] that are trained to estimate the position of key landmarks, such as the eye and the mouth region for our system. The ensemble is trained using a labeled dataset of images that specify the coordinates of the regions surrounding each facial feature. This landmark predictor enables fast and accurate detection in real-time.
The next step in determining the driver's fatigue level is based on analyzing the state of the facial landmarks that have been identified in each frame. To analyze the state of the eye, we consider the convex shape formed around the eye region and calculate its Eye Aspect Ratio, as illustrated in the previous section. If the EAR falls below Te, the eye is considered to be closed. Depending on the duration of eye closure, fatigue can be classified into three stages which will be discussed in the next section.

Similarly for the mouth, the Mouth Aspect Ratio is calculated. If this MAR exceeds Tm, the mouth is considered to be open in a state of yawning. Depending on the frequency of yawns, the system decides the level of fatigue and issues appropriate alerts.

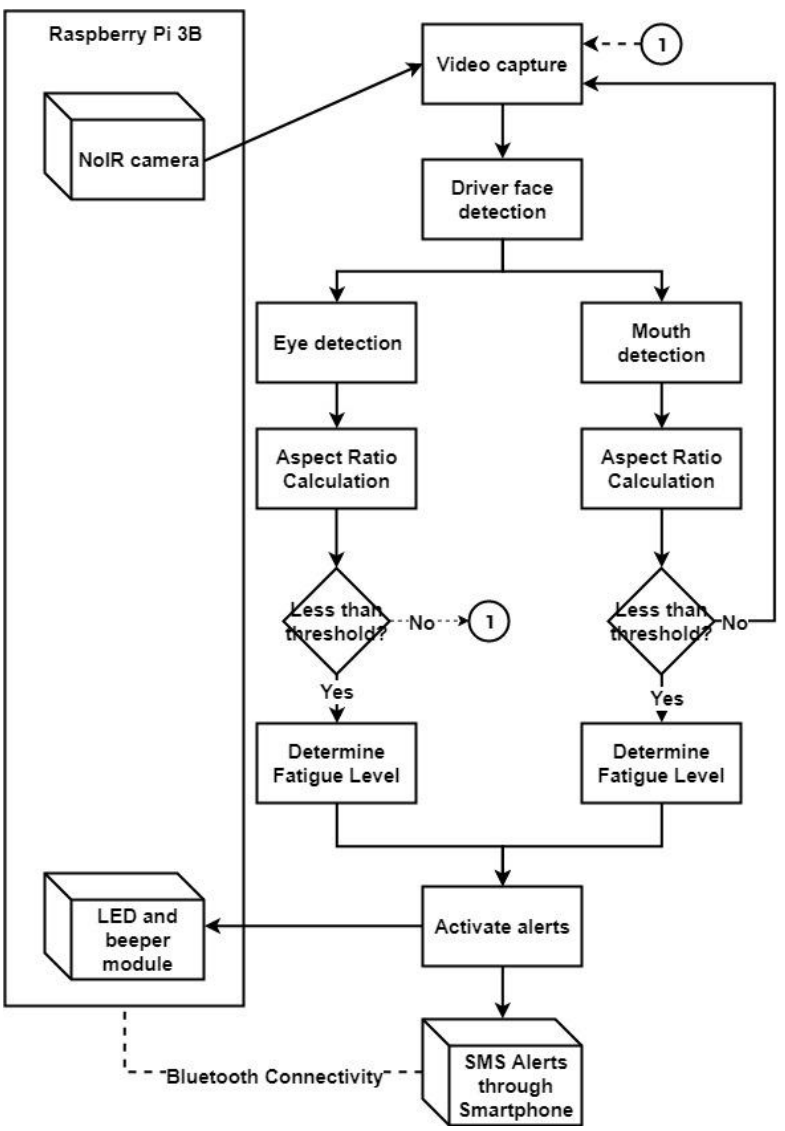

Fig 4: Control Flow Diagram of the System

\subsection{Fatigue Classification and Alerts}

Depending on the level and significance of drowsiness detected, the system classifies fatigue into three stages-

\subsubsection{Stage $1(\mathrm{~S} 1)$}

Stage 1 is the transition between wakefulness and sleep. In this stage, there is a shift in and out of consciousness, but it is easy to be awakened from this stage of sleep [12][13]. When Stage 1 of fatigue is detected, the driver is alerted by the means of a simple buzzer sound and bright LED blinking.

\subsubsection{Stage $2(S 2)$}

Stage 2 is a phase of faint sleep. During this stage of sleep, brain activity slows from waking levels and the driver may lose a sense of time and place. During both Stages 1 and 2, the body loses consciousness slowly as it prepares to fall into deeper phases of sleep [12]. In this stage, the system increases the intensity and frequency of audio and visual alerts through 
the buzzer and LED to make sure that he driver has been properly awakened.

\subsubsection{Stage 3 (S3)}

Stage 3 is the critical phase of the system where the driver actually falls asleep and loses consciousness. This stage is called the NREM sleep and can normally last between 1-10 minutes if left uninterrupted [14][15]. When Stage 3 is detected, the system issues highest level of local alerts by emitting loud, insistent beeping sound and flashing bright LEDs for catching the attention of the driver and alerting him to wake up and pull over. Apart from these local alerts, the system now connects to the driver's Android smartphone via Bluetooth and starts sending remote alerts as well.

Table 1.Fatigue Classification by Eye Closure

\begin{tabular}{|c|c|}
\hline Duration of Eye Closure & Fatigue Stage \\
\hline $1.5-2.5$ seconds & Stage 1 \\
\hline $2.5-3.5$ seconds & Stage 2 \\
\hline More than 3.5 seconds & Stage 3 \\
\hline
\end{tabular}

Table 2.Fatigue Classification by Yawn Frequency

\begin{tabular}{|c|c|}
\hline Time between Consecutive Yawns & Fatigue Stage \\
\hline 60 seconds & Stage 1 \\
\hline 30 seconds & Stage 2 \\
\hline
\end{tabular}

\subsection{Remote Alerts}

The system comes with the functionality of being able to send remote alerts to upto three contacts on the driver's Android smartphone in case of extreme fatigue. These settings are managed on the smartphone through a customized Android app. When Stage 3 of fatigue is detected, the Raspberry Pi module sends a signal to the smartphone. This signal is sent in the form of Bluetooth Low Energy (BLE) Beacons [16]. These beacons are activated by the Raspberry Pi module as soon as Stage 3 fatigue is encountered. The smartphone app starts listening for this signal on a BLE Scanner terminal as soon as it is paired with the Raspberry Pi module for the first time.

When the smartphone receives a Stage 3 signal, it sends remote SMS alerts to the driver's predefined contacts. This SMS message contains the current location of the driver, pinpointed through Android GPS, along with a short SOS message that informs the receiver of the driver's condition. These messages are sent three times, once in every five minutes, so that the receiver can get an estimate of the driver's exact location and direction of movement over time, in case the driver cannot be reached directly.

Since SMS alerts do well in alerting friends and family but not much in assisting the driver, the app also searches a $3 \mathrm{~km}$ radius around the driver's location for the nearest rest stops like hotels and cafes, and notifies the driver of the same.

\section{TESTING THE SYSTEM}

The Driver Fatigue Detection System is tested under varying conditions as may be experienced by a driver in real time. The factors considered while formulating test conditions include-

1. Lighting: Day and night time

2. Appearance: With and without prescription spectacles

The average frame rate of image capture during testing varied within the range of 6 to 8 FPS. Table III shows actual results obtained using the proposed system and Table IV shows the final interpretation of the tests performed on the system in terms of accuracy and precision in fatigue detection.

Table 3. Raw results from test runs

\begin{tabular}{|c|c|c|c|c|c|c|c|c|}
\hline \multirow{2}{*}{} & \multicolumn{5}{|c|}{ Eyes } & \multicolumn{2}{c|}{ Yawn } \\
\cline { 2 - 9 } & \multicolumn{3}{|c|}{ Actual } & \multicolumn{3}{c|}{ Detected } & \multirow{2}{*}{ Actual } & Detected \\
\cline { 2 - 9 } & S1 & S2 & S3 & S1 & S2 & S3 & & \\
\hline Test1 & 3 & 2 & 2 & 5 & 3 & 2 & 4 & 4 \\
\hline Test2 & 1 & 2 & 3 & 3 & 3 & 3 & 4 & 2 \\
\hline Test3 & 6 & 2 & 1 & 7 & 2 & 2 & 5 & 4 \\
\hline Test4 & 5 & 3 & 1 & 5 & 3 & 1 & 2 & 1 \\
\hline Test5 & 4 & 4 & 2 & 5 & 4 & 2 & 5 & 4 \\
\hline Test6 & 4 & 3 & 1 & 5 & 3 & 1 & 3 & 3 \\
\hline Test7 & 3 & 2 & 2 & 5 & 3 & 2 & 4 & 3 \\
\hline Test8 & 6 & 2 & 2 & 8 & 4 & 2 & 5 & 5 \\
\hline
\end{tabular}

Table 4.System performance calculated from raw results

\begin{tabular}{|c|c|c|c|c|}
\hline & \multicolumn{2}{|c|}{ Eyes } & \multicolumn{2}{c|}{ Yawn } \\
\cline { 2 - 5 } & Accuracy & Precision & Accuracy & Precision \\
\hline Test 1 & $100 \%$ & $70 \%$ & $100 \%$ & $100 \%$ \\
\hline Test 2 & $100 \%$ & $67 \%$ & $50 \%$ & $100 \%$ \\
\hline Test 3 & $100 \%$ & $82 \%$ & $80 \%$ & $100 \%$ \\
\hline Test 4 & $100 \%$ & $100 \%$ & $50 \%$ & $100 \%$ \\
\hline Test 5 & $100 \%$ & $91 \%$ & $80 \%$ & $100 \%$ \\
\hline Test 6 & $100 \%$ & $89 \%$ & $100 \%$ & $100 \%$ \\
\hline Test 7 & $100 \%$ & $70 \%$ & $75 \%$ & $100 \%$ \\
\hline Test 8 & $100 \%$ & $71 \%$ & $100 \%$ & $100 \%$ \\
\hline Average & $100 \%$ & $82 \%$ & $79 \%$ & $100 \%$ \\
\hline
\end{tabular}

The testing results show that the system performs quite well under the different conditions that have been considered. Night time has no significant adverse effect on the system due to the use of a NoIR camera and IR lamp. Use of spectacles by the driver also doesn't hinder the performance of the system as long as the eye region is not getting completely occluded (Fig 5(b)).

Some observations that can be made from the results in Table IV are-

1. Eye-based fatigue detection takes place always without fail, but suffers from the problem of detecting false positives thus reducing its precision. This may be due to the limitations of the Haar cascade used to identify the face region, which also suffers from the same problem (Fig 5(d)).

2. In the case of yawn detection, an actual yawn is not always detected properly. This may be due to the condition that the driver opens his/her mouth too wide while yawning such that the basic structure of the face is disturbed and not recognised by the facial landmark detector. This causes the accuracy of yawn detection to go down. 


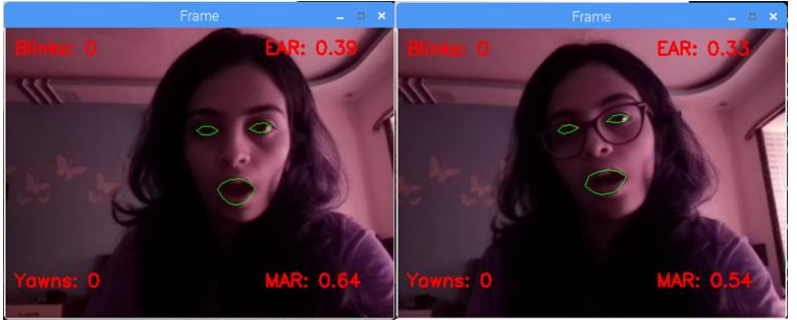

(a)

(b)

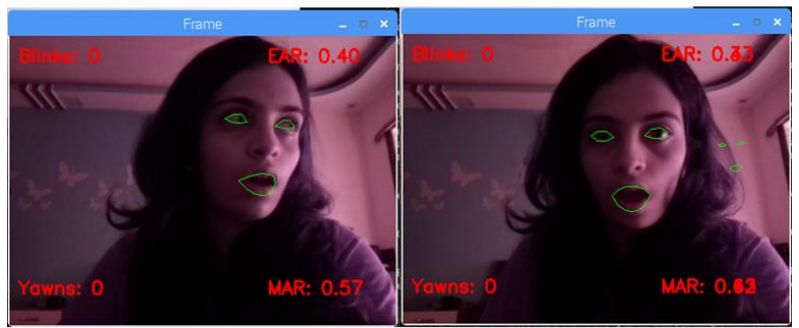

(c)

(d)

Fig 5: (a) Facial landmark detection under normal condition(b) Facial landmark detection with spectacles (c) Landmark detection when head faces away from camera (d) False Positive landmark detection due to limitations of Haar cascade

\section{CONCLUSION}

Our proposed system is developed using image processing to detect driver fatigue and set off alarms whenever necessary. The driver's video is captured and analyzed frame by frame on a real-time basis. The system is able to extract the face region and subsequently the required facial features using Haar cascade classifiers and an ensemble of regression trees. Fatigue is detected based on the extent of change in shape of these facial features. When fatigue is detected, the system issues local and remote alerts to awake the driver. Local alarms include components like LEDs and buzzers, whose frequency changes according level of fatigue detected. Remote alerts include customized SMS messages sent to the driver's emergency contacts along with resting solutions for the driver. Thus our system is reliable, effective and economical.The detection of fatigue from its very preliminary stages not only helps the system in issuing timely warnings, but also cautions the driver well in advance, potentially saving lives from unfortunate mishaps.

Although the system provides a lot of functionality, there is always scope for its betterment by involving higher levels of actions. By implementing the following suggestions, a Driver Fatigue Detection System can be made more robust and effective for commercial usage-

1. Integrating the system with the machinery of the vehicle itself. Allowing the system to control the vehicle's speed and indicator lights can help the alert the co-passengers of the drowsy driver as well as other vehicles in the vicinity.

2. Developing a system in partnership with the local department of road safety, like traffic police. When high level of fatigue is detected, the system may attempt to alert local road safety authorities who can immediately dispatch patrol vehicles.

\section{ACKNOWLEDGMENTS}

The authors also appreciate the support of the professors and assistants of Computer Engineering department, K.J. Somaiya College of Engineering for their guidance, feedback, and encouragement.

\section{REFERENCES}

[1] Transport Accident Commission. "Fatigue Statistics." Transport Accident Commission, TAC, 18 Oct. 2017, www.tac.vic.gov.au/roadsafety/statistics/summaries/fatigue-statistics.

[2] Dobbie, K. (2002). "An Analysis of Fatigue-related Crashes on Australian Roads using an Operational Definition of Fatigue", Australian Transport Safety Bureau, Road Safety Research Report OR 23.

[3] Krajewski, J., Sommer, D., Trutschel, U., Edwards, D., \& Golz, M. (2009). Steering wheel behavior based estimation of fatigue.

[4] Sahayadhas, A., Sundaraj, K., \& Murugappan, M. (2012). Detecting driver drowsiness based on sensors: a review. Sensors, 12(12), 16937-16953.

[5] Ingre M., ÅKerstedt T., Peters B., Anund A., Kecklund G. Subjective sleepiness, simulated driving performance and blink duration: Examining individual differences. J. Sleep Res. 2006;15:47-53.

[6] Akin M., Kurt M., Sezgin N., Bayram M. Estimating vigilance level by using EEG and EMG signals. Neural Comput. Appl. 2008;17:227-236.

[7] Linux, and Ben Martin. "Give Your Raspberry Pi Night Vision With the PiNoir Camera." Linux.com, 15 July 2015, www.linux.com/learn/give-your-raspberry-pinight-vision-pinoir-camera.

[8] Deza, Elena; Deza, Michel Marie (2009). Encyclopedia of Distances. Springer. p. 94.

[9] Tiesheng Wang and Pengfei Shi, "Yawning detection for determining driver drowsiness," Proceedings of 2005 IEEE International Workshop on VLSI Design and Video Technology, 2005., 2005, pp. 373-376.

[10] Viola, P., \& Jones, M. J. (2004). Robust real-time face detection. International journal of computer vision, 57(2), 137-154

[11] Kazemi, V., \& Josephine, S. (2014). One millisecond face alignment with an ensemble of regression trees. In 27th IEEE Conference on Computer Vision and Pattern Recognition, CVPR 2014, Columbus, United States, 23 June 2014 through 28 June 2014 (pp. 1867-1874). IEEE Computer Society

[12] "S+ ResMed." Sleep Architecture - The Shape of Your Night's sleep.mysplus.com/library/category3/article1.html.

[13] Wen-Bing Horng, Chih-Yuan Chen, Yi Chang and Chun-Hai Fan, "Driver fatigue detection based on eye tracking and dynamk, template matching," IEEE International Conference on Networking, Sensing and Control, 2004, Taipei, Taiwan, 2004, pp. 7-12.

[14] Felber, Susie. "The 4 Different Stages of Sleep." Nokia Health, Nokia Health Blog, 17 Mar. 2015, blog.health.nokia.com/blog/2015/03/17/the-4-differentstages-of-sleep/.

[15] K. U. Anjali, A. K. Thampi, A. Vijayaraman, M. F. Francis, N. J. James and B. K. Rajan, "Real-time nonintrusive monitoring and detection of eye blinking in view of accident prevention due to drowsiness," 2016 International Conference on Circuit, Power and Computing Technologies (ICCPCT), Nagercoil, 2016, pp. 1-6.

[16] Granbery, John Hastings. "Facilitating wireless connections using a BLE beacon." U.S. Patent No. 9,648,652. 9 May 2017. 